\title{
Macrophytes of Rajarani-Dhimal Pokhari Wetland, Churia hills, Eastern Nepal
}

\author{
Rajesh Chaudhary and Bhabindra Niroula* \\ Department of Botany, Post Graduate Campus, T.U., Biratnagar, Nepal \\ *E-mail: niroulab@gmail.com
}

\begin{abstract}
Growth forms, availability and uses of macrophytes found in Rajarani Dhimal Pokhari wetland, Churia hills, eastern Nepal was carried out in 2016. Altogether, 75 species belonging to 64 genera, 30 families, and 7 growth forms were recorded. Growth form classes were: helophytes and hyperhydates $(34.7 \%)>$ tenagophytes $(16 \%)>$ pleustophytes $(8 \%)$ rosulates, epihydates $(2.6 \%)>$ vittates (1.4\%). Nymphea nauchelii, Rorrippa indica; and Cephalanths tetrandra were new aquatic macrophytes to eastern Terai and Nepal, respectively. Aquatic macrophytes useful to local socioeconomy were: feed for livestock (17 sp), edible as pot herbs and wild fruits (5 sp), medicinal (19 sp), green manure/compost (7 sp), fish poison (2 $\mathrm{sp})$, handicrafts as mats/brooms/basketry $(8 \mathrm{sp})$, breeding genetic stock (2 $\mathrm{sp})$ and religious (3 sp). The key species of the wetland Cephalanhus tetrandra is under threat. of Mikania micrantha-an invasive alien species (IAS).
\end{abstract}

Key words: Cephalanths tetrandra, Growth forms, Helophytes, Invasive alien species

\section{Introduction}

Wetlands are one of the most productive ecosystems in the world and essential life supporting systems providing a wide array of benefits. They are valuable sources, sinks, and transformers of chemical, biological and genetic materials (Mitsch \& Gooselink, 2000). Aquatic macrophytes are natural biotic components whose abundance influences the structural and functional characteristics of wetland (Canfield et al., 1984). They dynamically guide cycle of minerals and other organic compounds, thereby influencing overall biomass production of water bodies and it can serve as indicator of monitoring the degree of damage in the ecosystem. Floristic list in a particular area gives reliable background information about the species diversity in community as each plant species has its own specific ecological amplitude and the same indicates the ecological nature of the habitat. There are reports on macrophytes of Terai region (Shrestha, 1996, Jha et al. 2005, Siwakoti, 2006, Niroula et al. 2010, Niroula \& Singh, 2010). Present communication enumerates the aquatic macrophytes, growth forms, use category and availability of macrophytes in the Raja-Rani Dhimal Pokhari wetland located at Churia hills.

\section{Materials and Methods}

Study area

Rajarani Dhimal Pokhari wetland ( $26^{\circ} 44.9^{\prime} 22^{\prime \prime} \mathrm{N}, 8^{\circ} 28.9^{\prime} 10^{\prime \prime} \mathrm{E}$; alt. $\left.470 \mathrm{~m} \mathrm{msl}\right)$ is situated in Churia hills at Letang-Bhogateni Municipality - 4, Morang district, Province 1, eastern Nepal. It lies within the Rajarani community forest (1700 ha) area. The wetland (133 ha) 
consists of Raja, Rani and Rajkumari Pokhari (20 ha). Depth of ponds ranges 2-7 m during rainy season and small Rajkumari Pokhari is almost dry in summer season. The wetland is culturally and religiously important for Dhimal community.

The climate of the wetland is subtropical monsoon type. The average annual minimum and maximum temperature ranges from $12-19{ }^{\circ} \mathrm{C}$, and $22-30{ }^{\circ} \mathrm{C}$, respectively. The total annual rainfall varies from $1,138 \mathrm{~mm}$ to $2,671 \mathrm{~mm}$ (FRA/DFRS, 2014).

An intensive survey of macrophytes was undertaken seasonally in the year 2016. Identification and growth form categories were determined by standard literature (Hooker, 1872-1897; Cook, 1996). The nomenclature of the plants was based on Hara et al. 19781982. Availability of the plants were noticed as abundant (++++), frequent $(+++)$, occasional (++) and scarce $(+)$ by visual observation. Use category of the plants was determined with the help of literature (Anonymous, 1948-1976; Banerjee, 1995; Bala \& Mukherjee, 2007; Majupuria \& Joshi, 2009).

\section{Results and Discussion}

Altogether 75 species of aquatic plants belonging to 64 genera and 30 families were recorded in the Rajarani Dhimal pokhari wetland. Dominant families in number of species were: Poaceae (12), Asteraceae (10), Cyperaceae (7), Araceae, Polygonaceae(4), Apiaceae, and Scrophulariaceae (3). Cephalanthus tetranda, Osbekia stellata and Pandanus nepalensis were woody perennials. Growth forms, availability and various uses of aquatic macrophytes occurring in the Rajarani Dhimal Pokhari wetland Letang-Bhogateni, Morang, eastern Nepal is given in Table 1. Nymphea nauchelii and Rorippa indica were new to Terai whlle Cephalanths tetrandra was new to Nepal in the Rajarani Dhimal Pokhari wetland. Unique natural habitat of the study site is favourable for luxuriant growth of aquatic vegetation. Dhimal Pokhari wetland exceeded in number of species occurring at Betana wetland Belbari, Singhia river and Bhattapokhari of Biratnagr, eastern Terai, Nepal (Niroula, 2011). Conspectus of aquatic species in the wetland is given in Table 2.

Table 1. List of macrophytes in Raja-Rani Dhimal Pokhari Wetland Letang-Bhogateni, Morang, Eastern Nepal.

\begin{tabular}{lllc}
\hline SN Name of species & L.N/C.N & $\begin{array}{l}\text { Gr. Use } \\
\text { F. cat. }\end{array}$ & Avail. \\
\hline $\begin{array}{l}\text { Bryophyta } \\
\text { Ricciaceae } \\
\text { Riccia fluitans L. }\end{array}$ & Leu & Ple - & + \\
\hline $\begin{array}{l}\text { Pteridophyta } \\
\text { Athyriaceae }\end{array}$ & & & \\
2. Diplazium esculentum (Retz.) Sw & Niguro & Hel F & ++ \\
$\begin{array}{l}\text { Azollaceae } \\
\text { 3. Azolla imbricata } \text { (Roxb.) Nakai }\end{array}$ & Pani uneu & Ple C & ++ \\
$\begin{array}{l}\text { Equisetaceae } \\
\text { 4. Equisetum diffusum } \text { D. Don }\end{array}$ & Kurkure & Hel M & ++ \\
$\begin{array}{l}\text { Thelypteridaceae } \\
\text { 5. Thelypteris dentata } \text { (Forsk) St. John }\end{array}$ & Niguro & Hyp - & +++ \\
6. T. prolifera (Retz.) C.F. Reed & Fern & Hel - & + \\
\hline
\end{tabular}




\section{Angiospermae \\ Dicotyledonae \\ Acanthaceae}

7. Hygrophila polysperma (Roxb.) T. Anders

8. H. quadrivalvis (Buch.-Ham.) Nees Amaranthaceae

9. Alternanthera sessilis (L.) DC

10 Celosia argentea L. Apiaceae

11. Centella asiatica (L.) Urb.

12. Hydrocotyle sibthorpioides Lamk.

13. Oenanthe javanica (Blume) DC Asteraceae

14. Adenostemma lavenia (L.) Kuntze

15. *Ageratum conyzoides $\mathrm{L}$.

16. *A. haustonianum Miller

17. Cotula hemispherica (Roxb.) Wall. ex. C.B. Clarke Gandha madini

18. Eclipta prostrata (L.) L.

19. Gnaphalium affine D.Don

20. G. polycaulon Pers.

21. Grangea maderaspatana (L.) Poiret

22. *Mikania micrantha Kunth.

23. Sphaeranthus indicus L.

24. Spilanthes iabadicensis A.H. moore Gentianaceae

25. Nymphoides hydrophyllum (Lour.) O. Kuntze Brassicaceae

26. Rorippa indica (L.) Hiern Lythraceae

27. Rotala rotundifolia (Buch.-Ham Roxb) ex D. Don Sim jhaar Melastomaceae

28. Osbekia stellata Buch.-Ham. ex D. Don Nympheaceae

29. Nymphea nauchelli N.L. Burman Onagraceae

30. Ludwigia octovalvis (Jacq. ) Raven

31. L. perennis L. Polygonaceae

32. Persicaria barbatum (L.) Hara

33. P. glabrum Willd

34. P. hydropiper L.

35. P. sp.

Rubiaceae

36. Cephalanthus tetrandra (Roxb.)

37. Hedyotis diffusa Willd

Scrophulariaceae

38. Lindernia oppositifolia (L.) Mukharjee

39. Limnophila rugosa (Roth) Merril

40. Mecardonia procumbens (Mill.) Small Urticaceae

41. Pouzolzia zelanica (L.) J. Bennett \& Brown

\begin{tabular}{|c|c|c|}
\hline Talmakhan & Нур M & ++ \\
\hline- & Нyр - & ++ \\
\hline Bhringi jhaar & Hel F & ++ \\
\hline Raswari saag & Hel - & ++ \\
\hline Ghodtapre & Hel M & ++ \\
\hline Sano ghodtapre & Hel - & ++ \\
\hline Pani dhaniya & Hyp F & ++ \\
\hline Pani jhaar & Нyр - & ++ \\
\hline Ilame jhaar & Hel M & ++ \\
\hline Nilo gandhe & Hel - & ++ \\
\hline Gandha madini & Hel C & ++ \\
\hline Bhringaraj & Hel M/R & ++ \\
\hline Booki phul & Hel - & ++ \\
\hline Bhuin booki & Hel - & ++ \\
\hline Gobre jhaar & Hel C & ++ \\
\hline Banmara & Hel - & ++ \\
\hline Bhuin kadam & Hel M & + \\
\hline Purpure & Ten $\mathrm{M}$ & ++ \\
\hline Floating heart & Eph M & ++ \\
\hline Yellow cress & Ten - & ++ \\
\hline Sim jhaar & Нур - & ++++ \\
\hline Angeri & Hel F & ++ \\
\hline Kamal & Eph R & +++ \\
\hline Luwange & Нyp - & +++ \\
\hline Luwange & Нyp M & ++ \\
\hline Pirre jhaar & Нур M & ++ \\
\hline Pirre jhaar & Нyp M & ++ \\
\hline Pirre jhaar & Hyp FP & ++ \\
\hline Pirre Jhaar & Hyp FP & ++ \\
\hline Pani simali & Нyp - & ++++ \\
\hline Majithe jhaar & Hel - & ++ \\
\hline- & Ten - & ++ \\
\hline- & Ten - & ++ \\
\hline Baby jump up & Hel - & ++ \\
\hline Barbere & Hel - & ++ \\
\hline
\end{tabular}




\section{Monocotyledonae}

Aponogetonaceae Araceae

43. Acorus calamus L.

44. Colocasia esculenta (L.) Schott.

45. Lasia spinosa (L.) Thwaites

46. Pistia stratioites L. Commelinaceae

47. Commelina paludosa Blume

48. Floscopa scandens Lour.

49. Murdania vaginata (L.) Bruckn Cyperaceae

50. Carex nubigena D. Don

51. Cyperus amabilis Vahl

52. C. esculentus L.

53. C. pilosus Vahl

54. C. platystylis $\mathrm{R}$. Br.

55. Fimbristylis dichotoma (L.) Vahl.

56. Kyllinga brevifolia Rottb. Hydrocharitaceae

57. Ottelia alismoides (L.) Pers. Lemnaceae

58. Lemna minor L.

59. Spirodela polyrhiza (L.) Schleiden Najadaceae

60. Najas graminea Dulile Pandanaceae

61. Pandanus nepalensis St. John Poaceae

62. Arundinella nepalensis Trin.

63. Cynodon dactylon (L.) Pers

64. Digitaria setigera Roth apud Roem. \& Schultz.

65. Hemarthria compressa (L. f.) R. Br

66. *Leersia hexandra Swartz

67. Oplismenus composites (L.) Beauvois

68. Panicum psilopodium Roxb.

69. Paspalum distichum $\mathrm{L}$.

70. Phragmites karka (Retz.) Trin ex. Steudel

71. Saccharum spontaneum L.

72. Sacciolepis indica (L.) Chase

73. Setaria paludefusca Schumah Pontederiaceae

74. *Eichhornia crassipes (mart.) Solms. Typhaceae

75. Typha elephantina Roxb.
42. Aponogeton appendiculatus $\mathrm{H}$. Bruggen

\begin{tabular}{|c|c|c|}
\hline- & Ros - & ++ \\
\hline Bojho & Ten $\mathbf{M}$ & + \\
\hline Kachhu & Ten FO & + \\
\hline Morange saag & Hyp F & ++ \\
\hline Pani banda & Ple $\mathrm{C} / \mathrm{M}$ & ++ \\
\hline Swamp day flower & Hel - & ++ \\
\hline Kane & Hyp FO/M & +++ \\
\hline- & Ten FO & ++ \\
\hline Hatkatta & Hyp FO & ++ \\
\hline Mothe & Hyp HC & +++ \\
\hline Mothe & Нyp HC & ++ \\
\hline Keshar & Hyp HC & +++ \\
\hline Mothe & Нyp HC & ++ \\
\hline Mothe & Hyp FO & ++ \\
\hline Mothe & Hel FO & ++ \\
\hline Hile jhaar & Ros $\mathrm{FO} / \mathrm{M}$ & ++++ \\
\hline Duckweed & Ple C/M & ++ \\
\hline Duckweed & Ple C & ++ \\
\hline Water nymph & Vit - & + \\
\hline Dandi kath & Ten $\mathrm{M} / \mathrm{R} / \mathrm{HC}$ & ++++ \\
\hline River grass & Hyp FO & ++ \\
\hline Dubo & Hel M/FO/R & ++ \\
\hline Banso & Hel FO & + \\
\hline Ghode dubo & Ten FO & ++ \\
\hline Karaute & Hyp FO/GR & ++ \\
\hline- & Hel FO & ++ \\
\hline Janai ghans & Hyp FO & ++++ \\
\hline Panighans & Ten FO & + \\
\hline Narkat & Нyp HC & ++ \\
\hline Kans & $\mathrm{Hel} \mathrm{M} / \mathrm{FO} / \mathrm{GR} / \mathrm{HC}$ & ++ \\
\hline Panighans & Hyp FO & ++++ \\
\hline Ghoge banso & Ten FO & ++ \\
\hline Jalkumbhi & Ple C & ++++ \\
\hline Pater & Ten HC & ++ \\
\hline
\end{tabular}

$($ Hel = helophytes, Ten = tenagophytes, Hyp = hyperhydates, Eph = epihydates, Ple = pleustphytes, Ros $=$ rosulates, Vit $=$ vittates $; \mathrm{C}=$ compost manure, $\mathrm{F}=$ food, $\mathrm{FO}=$ fodder, $\mathrm{FP}=$ fish poison, $\mathrm{GR}=$ genetic resource, $\mathrm{HC}=$ handicrafts, $\mathrm{M}=$ medicinal, $\mathrm{R}=$ religious; $+=$ scarce, $++=$ occasional, +++ $=$ frequent, $++++=$ abundant $) *$ Invasive alien species (IAS) 
Table 2. Conspectus of macrophytes occurring in Rajarani Dhimal Pokhari, Churia hills eastern Nepal.

\begin{tabular}{lccc}
\hline Taxa & Family & Genera & Species \\
\hline Bryophyta & 1 & 1 & 1 \\
Pteridophyta & 4 & 4 & 5 \\
Angiosperm-Dicots & 14 & 28 & 35 \\
Angiosperm- & 11 & 31 & 34 \\
Monocots & 30 & 64 & 75 \\
\hline Total & &
\end{tabular}

Eichhornia crassipes, ottelia alismoides, Panicum psilopodium, Rotala rotundifolia and Sacciolepis indica were abundant while Acorus calamus, Arundinella nepalensis, Digitaria setigera, Paspalum distichum and Najas graminea were scarce. Ageratum conyzoides, A. haustonianum, E. crassipes, and Mikania micrantha were invasive alien species of the wetland. The key species of the wetland Cephalanhus tetrandra is under threat by $M$. micrantha. Invasive alien species form part of the vegetation in the disturbed areas and they are most susceptible to biological invasion (Mitchell \& Gopal, 1991).

Seven growth forms of aquatic plants were recorded in the Raja-Rani Dhimal Pokhari wetland. Percentage contribution to each growth form classes were in the order: Helophytes and Hyperhydates (34.7\%) > Tenagophytes (16\%) > Pleustophytes (8\%) Rosulates, Epihydates (2.6\%) > Vittates (1.4\%).

Aquatic macrophytes were found to play substantial role in local socio-economy; feed for livestock (17 sp.), edible as pot herbs and wild fruits (5 sp.), medicinal (19 sp.), green manure/compost (7 sp.), fish poison ( $2 \mathrm{sp}$.), handicrafts as mats/brooms/basketry ( $8 \mathrm{sp}$.) and religious (3 sp.). Some plant had multiple uses. Miscellaneous uses of the macrophytes in the Rajarani Dhimal Pohhari wetland included as insect repellent-Acorus calamus and Sphaeranthus indicus; dyes-Eclipta prostrate and Persicaria hydropiper; breeding genetic stocks for rice- Leersia hexandra and sugarcane- Saccharum spontaneum.

\section{Acknowledgement}

The first author is thankful to the Head, Department of Botany, Post Graduate Campus, T.U., Biratnagar, Nepal for providing laboratory facilities.

\section{References}

Anonymous 1948-1976. Wealth of India: raw materials (I-X). CSIR, New Delhi.

Bala, G. \& A. Mukhrjee 2007. Useful plants of wetland in Nadia district, West Bengal. Geobios 34(4): 253-256.

Banerjee, M.L. 1995. Some edible and medicinal plants from east Nepal. J. Bomb. Nat. Hist. Soc. 53: 153-155.

Canfield, D.E., J.V. Shirman \& J.R. Jones 1984. Assesing the trophic status of lakes with aquatic macrophytes. Lakes and Reserv. Manag. 1: 446-450.

Cook, C.D.K. 1996. Aquatic and Wetland Plants of India. Oxford University Press, Oxford, UK. 
FRA/DFRS 2014. Churia forests of Nepal (2011-2013). Forest Resource Assessment of Nepal Project and Department of Forest Research and Survey. Babarmahal Kathmandu.

Hara, H. \& L.H. Williams 1979. An enumeration of the flowering plants of Nepal, vol. 2. British Museum (Nat. Hist.), London, UK.

Hara, H., A.O. Chater \& L.H. Williams 1982. An enumeration of the flowering plants of Nepal, vol. 3. British Museum (Nat. Hist.), London, UK.

Hara, H., W.T. Stearn and L.H. Williams 1978. An enumeration of the flowering plants of Nepal, vol. 1. British Museum (Nat. Hist.), London, UK.

Hooker, J.D. 1872-1897. The flora of British India, vol. 7. L. Reeve, London.

Jha, S., U. Koirala \& B. Niroula 2005. Plant resources of Betana Taal and adjoining areas. Report submitted to Association for Protection of Environment and Culture (APEC), Biratnagar.

Majupuria, T.C. \& D.P. Joshi 2009. Religious and useful plants of Nepal and India. Craftsman Press Ltd. Bankok, Thailand.

Mistch, W.J. \& J.G. Gosselink 2000. Wetlands. John Wiley and Sons, Inc. New York, Chichester, Weinhelm, Brisbane, Singapore, Toronto.

Mitchell, D.S. \& B. Gopal 1991. Invasion of tropical freshwater by alien aquatic plants. In: Ecolgy of Biological Invasion (Ed. P.S. Ramakrishnan). Elsevier, New York. pp. 139154.

MoFSC 2012. Nepal biodiversity strategy. Government of Nepal, Ministry of Forests and Soil Conservation, Nepal.

Niroula, B \& K.L.B. Singh 2010. Contribution of aquatic macrophytes of Biratnagar and adjoining areas, eastern Nepal. Ecoprint 17: 23-34.

Niroula, B. 2011. Ecology and phytosociology of aquatic macrophytes in and around Biratnagar, eastern Nepal. Ph. D Thesis. T.M. Bhagalpur University, India.

Niroula, B., K.L.B. Singh \& Aloka Kumari 2011. Seasonal occurrence of aquatic macrophytes in Singhiya river, Biratnagar, Nepal. Pragyik 2: 100-103.

Niroula, B., K.L.B. Singh, G.B. Thapa \& J. Pal 2010. Seasonal variations in physico-chemical properties and biodiversity in Betana Pond, eastern Nepal. Our Nature 8: 212-218.

Shrestha, P. 1996. Diversity of aquatic macrophytes in the Koshi Tappu Wildlife Reserve and surrounding areas, eastern Nepal. In: Environment and biodiversity in the context of south Asia. (Eds. P.K. Jha, G.P.S. Ghimire, S.B. Karmacharya and P. Lacoul). Ecological Society (ECOS), Kathmandu. pp. 203-211.

Siwakoti, M. 2006. An overview of floral diversity of wetlands of Tarai region of Nepal. Our Nature 4: 83-90. 